\title{
A Monte Carlo study of non-trapped self-avoiding walks
}

\author{
Yao-ban Chan ${ }^{1}$ and Andrew Rechnitzer ${ }^{2}$ \\ ${ }^{1}$ Fakultät für Mathematik, Universität Wien \\ 1090 Vienna, Austriał \\ E-mail: yao-ban.chan@univie.ac.at \\ 2 Department of Mathematics, University of British Columbia \\ Vancouver, Canada \\ E-mail: andrewr@math.ubc.ca
}

\begin{abstract}
In this paper, we study non-trapped self-avoiding walks, which are selfavoiding walks which can be extended to an infinite length. We apply the flatPERM Monte Carlo method for this purpose, generating non-trapped SAWs of length up to 1024 on the square, triangular and hexagonal lattices, and calculating their number and mean squared end-to-end distance. We find strong evidence that the growth constant $\mu$ and entropic and metric exponents $\gamma$ and $\nu$ are identical for non-trapped and all SAWs, and in particular that the exponents are also universal for non-trapped SAWs. We also calculate the limiting ratio of non-trapped to all self-avoiding walks. We see some evidence of a $n^{-1 / 2}$ correction-to-scaling term which is not present for all SAWs.
\end{abstract}

PACS numbers: 05.10.Ln, 05.40.Fb, 02.10.Ox, 02.50.Ng, 02.70.Uu

Submitted to: J. Phys. A: Math. Theor.

$\ddagger$ Some of this work was carried out at LaBRI, Université Bordeaux 1. 


\section{Introduction}

A self-avoiding walk (SAW) is a walk on the vertices of a regular lattice that never returns to a vertex that it has already visited. In this paper we are primarily concerned with SAWs on $\mathbb{Z}^{2}$, but also on the triangular and hexagonal lattices. The question of how many such walks of length $n$ there are is an open question which has been very well-studied (see for example $[1,2,3]$ ). In particular, the number of SAWs of length $n$ has the asymptotic behaviour

$$
c_{n} \sim A \mu^{n} n^{\gamma-1},
$$

where $\mu$ is not known, but for the square lattice it is estimated to be $\mu \approx$ $2.63815853031 \ldots$. [4], while $\gamma$ is known (but not proved) to be 43/32. Another quantity of interest is the mean squared end-to-end distance, which is the average over all SAWs of length $n$ of the squared distance between the endpoints of the walk. This quantity is believed to obey a power law

$$
\left\langle R^{2}\right\rangle_{n} \sim \alpha n^{2 \nu}
$$

where $\nu=3 / 4$; this exponent value is widely accepted, but again not proved.

A way of gaining insight into the asymptotic properties of self-avoiding walks is to observe the behaviour of certain restricted subsets of these walks. Examples include directed walks (walks which can only step north and east) and partially directed walks (walks which cannot step west), which are easily solvable but less interesting than larger subsets. More recently prudent self-avoiding walks — walks which can never step in the direction of a previously visited vertex — have been studied closely [5]. However, the growth constants of all of these models are strictly smaller than those of SAWs and so they are exponentially rare in the set of all SAWs.

In this paper, we examine a large subset of self-avoiding walks, non-trapped selfavoiding walks (NTSAWs). We define a NTSAW to be a SAW that can always be extended to an infinitely long SAW - that is, as the walk is grown from a single vertex, it is forbidden to enter a cul-de-sac from which it cannot leave. This is illustrated in figure 1. The main aim of this paper is to examine the scaling of such walks. To this end, we compute the number of non-trapped SAWs and their mean squared endto-end distance; we distinguish these values from the SAW values by using a a subor superscript "NT". This idea arose from a paper by Bousquet-Mélou [6] (see also [7]), which studied the Rosenbluth method on walks which cross a square, which are non-trapped by definition.

One can quickly show that NTSAWs have the same exponential growth rate as all SAWs. Since NTSAWs are a subset of SAWs, we must have $\mu_{N T} \leq \mu$. Then we introduce a subset of NTSAWs, bridges, to give the needed lower bound. A bridge is a SAW which starts on the left-hand side of its bounding box and ends on the right, with no other vertex on the left-hand side (see figure 1(c)). Bridges appear in many places in the literature of SAWs because their growth rate is the same as that of all SAWs $[8,9,3]$. Since all bridges are non-trapped, this implies that $\mu_{N T}=\mu$. It is far 


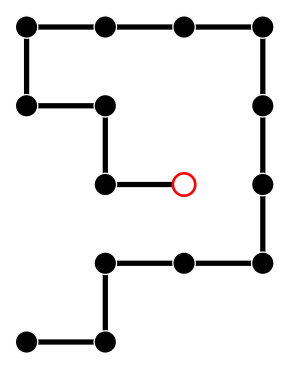

(a) A trapped walk

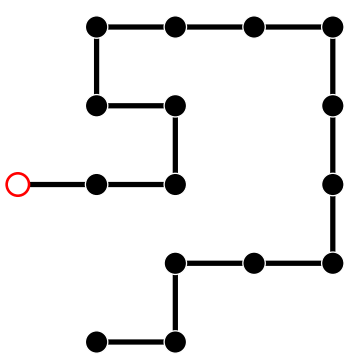

(b) A non-trapped walk

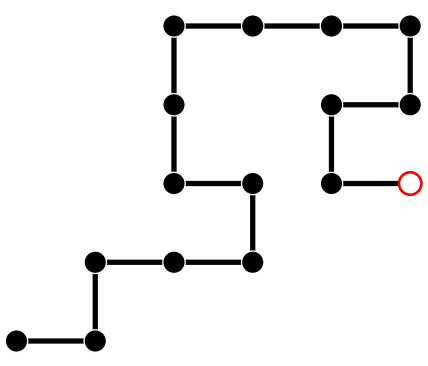

(c) A bridge

Figure 1. Trapped walks, non-trapped walks and bridges. The hollow vertex is the final vertex of the walk.

less obvious, however, whether or not $\gamma_{N T}=\gamma$. Now consider the end-to-end distance of NTSAWs; one might expect non-trapped walks to be "less compact" than regular SAWs and so have greater end-to-end distance. Again, it is natural to ask whether or not $\nu_{N T}=\nu$.

The main aim of this paper is to demonstrate that these exponents are equal namely that SAWs and NTSAWs have the same entropic and metric exponents. While $\mu$ varies with the underlying lattice, the exponents, $\gamma$ and $\nu$, are thought to be universal - depending only on the dimension of the lattice and not the details of its structure. To see if the corresponding quantities for NTSAWs share this property, we also consider them on the triangular and honeycomb lattices.

Note that an alternative, but non-equivalent, definition of non-trapped walks is a walk which cannot be extended by even a single step (i.e. every neighbour of the endpoint is occupied). If we use this definition, it has indeed been shown [10] that non-trapped walks have the same entropic exponent $\gamma$ as all SAWs.

A non-rigorous argument (due to Guttmann), which applies equally to both definitions of non-trapped walks, implies that if this is the case, then the metric exponent $\nu$ is identical. It is accepted [1] that the squared end-to-end distance of self-avoiding walks grows as

$$
\left\langle R^{2}\right\rangle_{n}=a n^{3 / 2}+b n^{1 / 2}+o\left(n^{1 / 2}\right) .
$$

Now

$$
\left\langle R^{2}\right\rangle_{n}=\frac{c_{n}^{N T}}{c_{n}}\left\langle R^{2}\right\rangle_{n}^{N T}+\frac{c_{n}^{T}}{c_{n}}\left\langle R^{2}\right\rangle_{n}^{T},
$$

where we use the " $T$ " superscript to denote trapped walks. If $\gamma_{N T}=\gamma$, then the ratio $c_{n}^{N T} / c_{n}$ converges to a limit between 0 and 1 . This must also be true for the ratio $c_{n}^{T} / c_{n}$ (for either definition of trapping); one can simply add a finite-length trapping configuration — such as a short spiral of bonds - to the end of any self-avoiding walk to make it trapped. Therefore one of $\left\langle R^{2}\right\rangle_{n}^{N T}$ and $\left\langle R^{2}\right\rangle_{n}^{T}$ must contribute the asymptotic term of $n^{3 / 2}$ to $\left\langle R^{2}\right\rangle_{n}$. Moreover, the other must either contribute the same asymptotic term or a term of $O\left(n^{1 / 2}\right)$. As a random walk with no restrictions has a mean squared 
end-to-end distance which grows like $n^{1}$, it follows that both terms must grow like $n^{3 / 2}$, and therefore $\nu_{N T}=\nu=3 / 4$.

In this paper, we use a Monte Carlo method, flatPERM [11], to analyse the asymptotic behaviour of non-trapped SAWs. In section 2 we describe this algorithm and the adaptations we have included to generate non-trapped SAWs. We analyse the results in section 3 and conclude in section 4 .

\section{Algorithm}

\subsection{FlatPERM}

To generate SAWs and NTSAWs, we use the flatPERM algorithm [11], and we start by giving a brief overview of it. This algorithm enhances the Rosenbluth method [12, 13], which is a kinetic growth algorithm which grows walks from a single point. At every step, the walk is extended at random to one of the unoccupied neighbours of its endpoint. If the endpoint has no unoccupied neighbours, the entire walk is discarded. This continues until the walk reaches a desired length, $n$ say. We start this growing process a specified number of times (rather than generating a specified number of valid walks).

This does not generate all self-avoiding walks with uniform probability; the probability of a specific walk, $\varphi$, of length $n$ being generated is

$$
\operatorname{Pr}(\varphi)=\prod_{k=0}^{n-1} a_{k}(\varphi)^{-1}
$$

where $a_{k}(\varphi)$ (the atmosphere of the walk at length $k$ ) is the number of valid ways that an edge can be appended to the walk's endpoint when the walk is of length $k$. Note that for SAWs the atmosphere is simply the number of unoccupied neighbours of the endpoint, while for NTSAWs it is more complicated (see below). To counter this uneven probability, we weight each walk by the inverse of its probability. The average weight of walks generated in this way is then simply the number of walks of length $n$

$$
\left\langle w_{n}\right\rangle=\sum_{\varphi} \operatorname{Pr}(\varphi) \cdot \operatorname{Pr}(\varphi)^{-1}=c_{n} .
$$

In practice, the Rosenbluth algorithm becomes ineffective at moderate length or longer, because the variance of probabilities of walks at the same length is very high [6]. Many walks of low weight and high probability are generated, but then occasionally an unlikely walk with large weight is sampled and swamps the statistics. The pruned and enriched Rosenbluth method, PERM, counters this by either making copies of partial walks with high weight, or eliminating copies of walks with low weight [14]. In either case the weight is then "normalised", which reduces the variance. We use a variant of the original PERM algorithm called flatPERM [11].

In this version, at length $k$ we keep an estimate of the number of walks $c_{k}^{\text {est }}$, and as each walk is grown we evaluate (at step $k$ ) the quantity $r=w_{k} / c_{k}^{e s t}$. Then:

- if $r>1$, we make $c=\min \left(\lfloor r\rfloor, a_{k}\right)$ copies of the walk, each with weight $w_{k} / c$, and grow each independently (enriching); or 


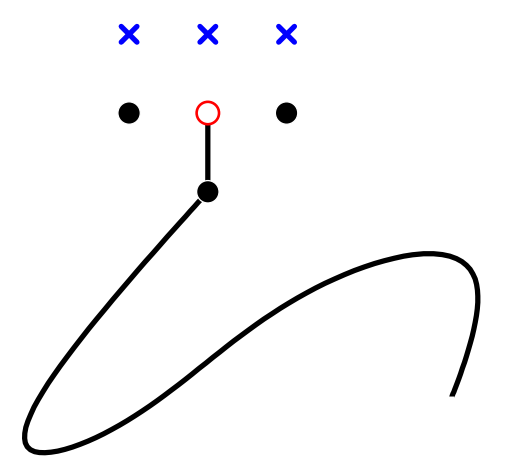

Figure 2. The three marked vertices must be checked to see if the walk will trap itself.

- if $r<1$, we either eliminate it with probability $1-r$, or continue growing it with weight $c_{k}^{e s t}$ (pruning).

The decision to prune or enrich is only calculated after the current walk is used to update $c_{k}^{e s t}$. Neither of these options changes the average of the weights, but they do reduce the variance.

\subsection{Non-trapped walks}

To generate non-trapped SAWs, we make a small alteration to this algorithm, suggested to us by Bousquet-Mélou [6]. Instead of choosing an unoccupied neighbour at random to extend the walk to, we choose a neighbour at random among all unoccupied neighbours whose addition would leave the walk untrapped. We adjust the definition of atmosphere for NTSAWs accordingly. Consider figure 2. To determine if the addition of a particular vertex would leave the walk untrapped, we check if any of the two diagonally opposite vertices or the directly opposite vertex from the last step are occupied. If one is, we observe in which way the walk winds around to the endpoint.

Take for example figure 3: in 3(a), the walk winds anti-clockwise to the endpoint, so an extension to vertex $a$ will trap the walk. On the other hand, in 3(b), the walk winds clockwise to the endpoint, so an extension to vertices $b$ or $c$ will trap the walk. Consequently we keep track of the winding number of the walk at each vertex. This is a number which starts at 0 , increases by 1 if the walk makes a right turn, and decreases by 1 if the walk makes a left turn. If the winding number of the occupied vertex is less than the winding number of the current vertex, then the walk winds clockwise; otherwise, it winds anti-clockwise. (Note that the winding numbers can never be equal in this context.) These updates and checks can be performed in constant time.

We note that because we are choosing only neighbours which do not trap the walk, we never have to eliminate a partial walk because it cannot grow further. Such a situation is common when generating regular self-avoiding walks, so this is one advantage that simulating non-trapped walks has over simulating all walks with the Rosenbluth method and its relatives. 


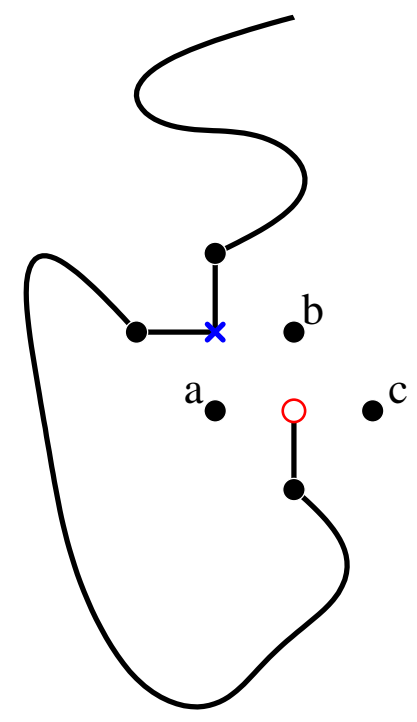

(a) Extension to a traps the walk

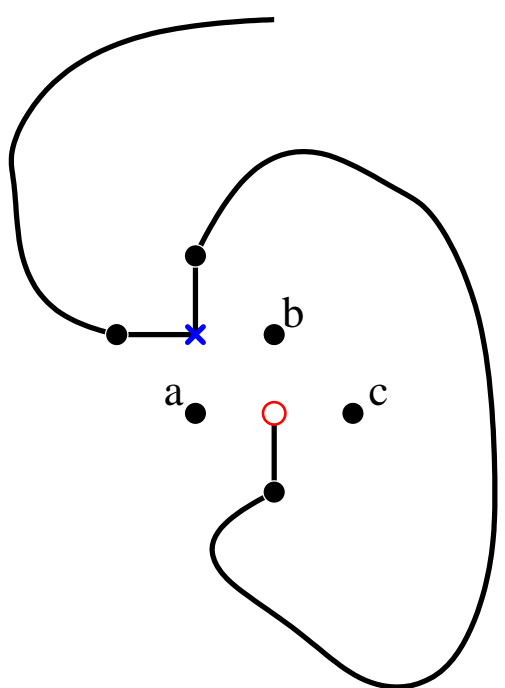

(b) Extension to $b$ or $c$ traps the walk

Figure 3. Depending on the wind of the walk, different vertices are forbidden.

\subsection{Other lattices}

The algorithm is readily adapted to generate walks on the triangular and honeycomb lattices. The only modification we need is to know which vertices to check to see if potential extensions will trap the walk. This is shown in figure 4. Unfortunately, checking for trapping in higher-dimensional lattices is considerably more complicated and computationally expensive and we have not pursued it here.

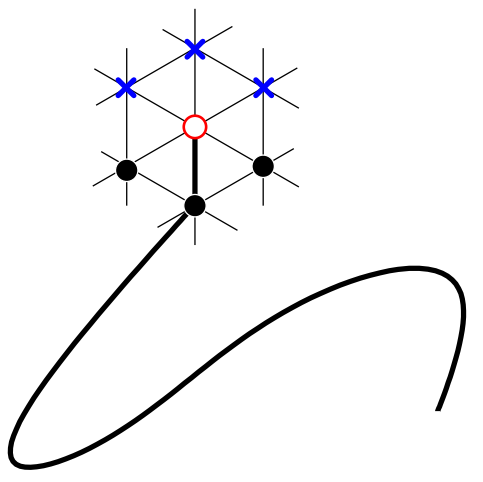

(a) Triangular lattice

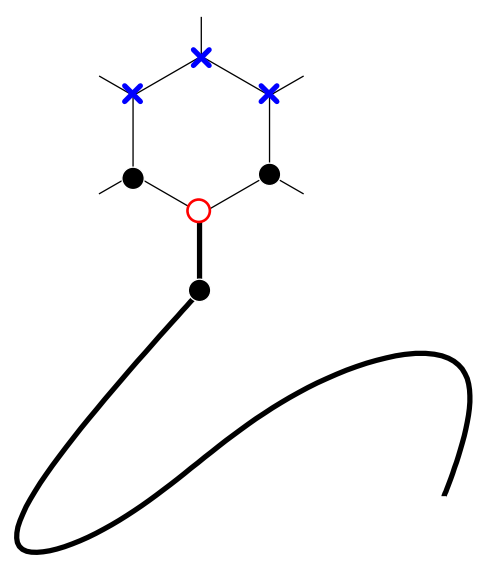

(b) Honeycomb lattice

Figure 4. Points to check for trapping in the triangular and honeycomb lattices. 


\section{Results}

We ran the algorithm to generate walks, both NTSAWs and SAWs, of length up to 1024 on each lattice. In each of these 6 cases, we ran 16 independent simulations for 1 week on the WestGrid super-computing cluster. Each node of the cluster uses $3.06 \mathrm{GHz}$ Intel Xeon 32-bit processors and 2GB of RAM. Due to slight differences in the algorithms on each lattice, we were able to generate between $2.7 \times 10^{8}$ and $4.1 \times 10^{8}$ walks. We then took averages of the data in each batch of 16 runs; the variance in this data also gave us an estimate of the statistical error. The resulting data are analysed below.

\subsection{Entropic and metric exponents}

Firstly, we estimate the value of $\gamma_{N T}$. We examine the square lattice first. Assuming the same asymptotic form for NTSAWs as for all SAWs, we anticipate a straight line fit to a $\log -\log$ plot of $c_{n}^{N T} / \mu^{n}$ against $n$, with a slope of $\gamma_{N T}-1$. Figure 5 clearly shows this to be the case. However, a simple linear fit to these points is not very accurate - if we fit a line to the range $n \in\left[n_{0}, \infty\right)$, then the estimate of $\gamma_{N T}$ shows a definite trend as $n_{0}$ increases (see figure 6). This is most likely due to sub-dominant terms in the asymptotic expansion.

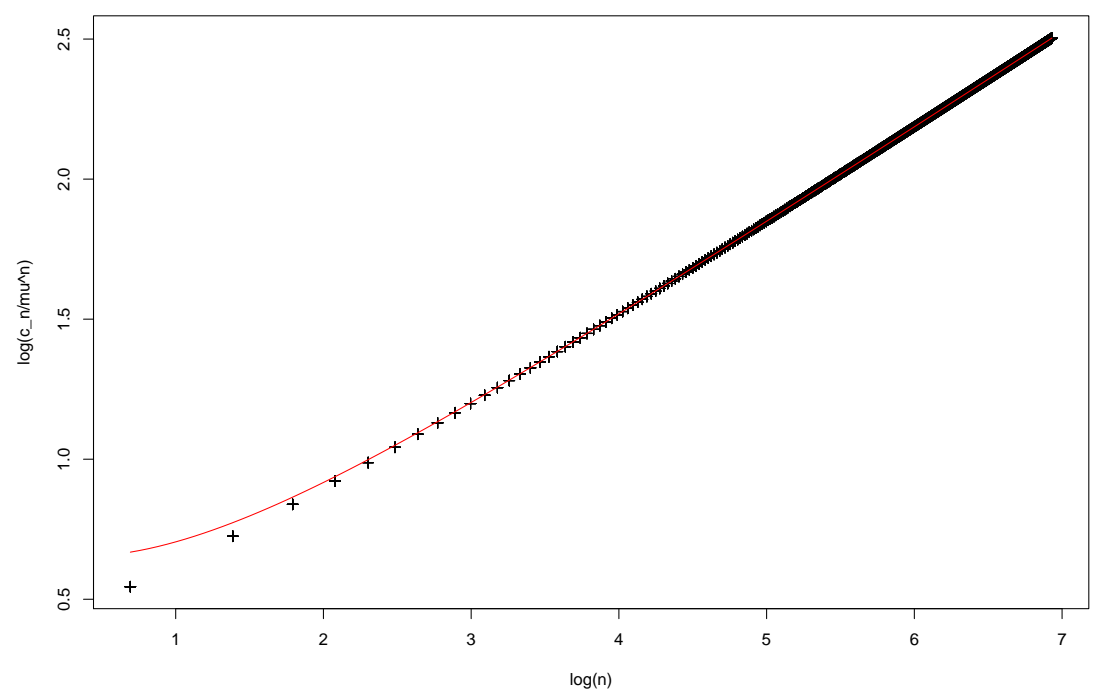

Figure 5. Log-log plot of $c_{n}^{N T} / \mu^{n}$ against $n$ for the square lattice, with fitted values using a model containing all terms with $n_{0}=100$.

A more accurate approximation comes from including the first (analytic) correctionto-scaling term for all self-avoiding walks [1]

$$
c_{n}=A \mu^{n} n^{\gamma-1}\left[1+\frac{B}{n}+O\left(\frac{1}{n^{3 / 2}}\right)\right],
$$




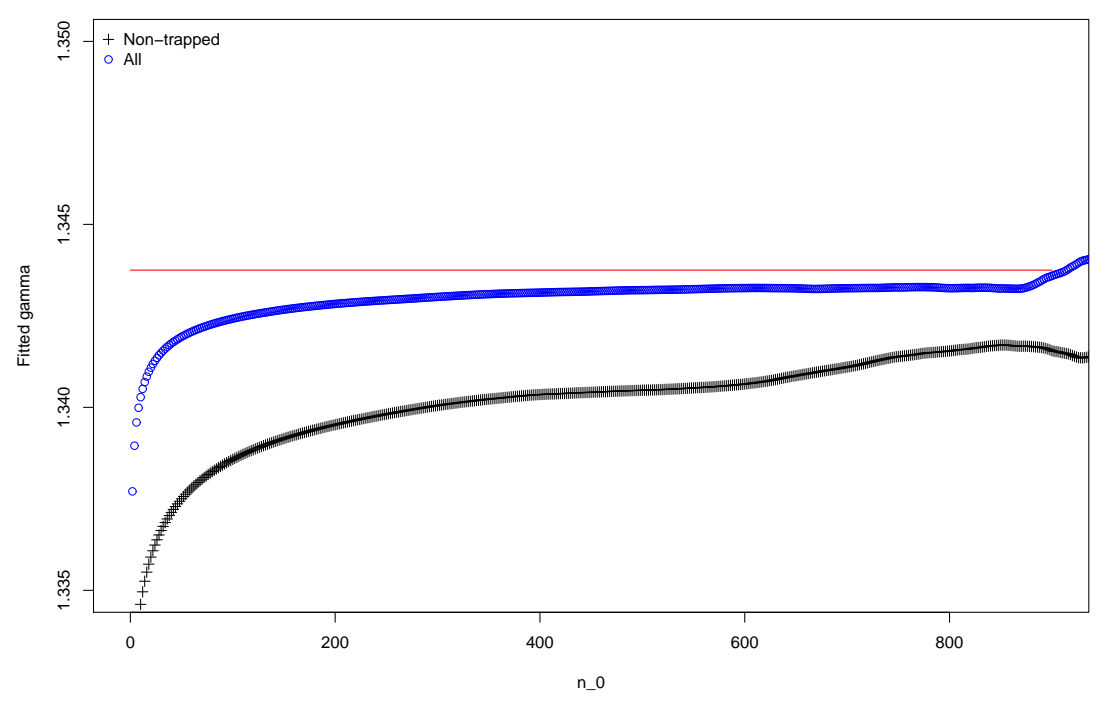

Figure 6. Fitted $\gamma_{N T}$ against $n_{0}$ for the square lattice, using simple linear fits over a fitting window of $\left[n_{0}, \infty\right)$. The other lattices are similar.

Table 1. Estimates of $\gamma, \gamma_{N T}, \nu$ and $\nu_{N T}$ for various lattices. The numbers in brackets are the standard error of our estimates. The true values of the parameters for SAWs are $\gamma=43 / 32=1.34375$ and $\nu=3 / 4$.

\begin{tabular}{lrrrl}
\hline Lattice & $\gamma$ & $\gamma_{N T}$ & $\nu$ & \multicolumn{1}{c}{$\nu_{N T}$} \\
\hline Square & $1.3439(4)$ & $1.3423(4)$ & $0.74990(10)$ & $0.74904(9)$ \\
Triangular & $1.3431(5)$ & $1.3427(4)$ & $0.74984(15)$ & $0.74907(15)$ \\
Hexagonal & $1.3439(3)$ & $1.3423(4)$ & $0.74985(12)$ & $0.74872(7)$ \\
\hline
\end{tabular}

which implies

$$
\log \frac{c_{n}}{\mu^{n}}=\log A+(\gamma-1) \log n+\frac{B}{n}+O\left(\frac{1}{n^{3 / 2}}\right) .
$$

Introducing an additional term of $1 / n$ corrects some of the systematic error in the fit, though it requires more data to be accurate (see figure 7 ). To estimate $\gamma_{N T}$ (and $\gamma$, as a check on our methods), we averaged the estimates in a range where they visually appear stable $-n_{0} \in[100,400]$ for NTSAWs and $n_{0} \in[50,400]$ for all SAWs. The results, along with those for the other lattices where we used a similar procedure, are shown in table 1.

It can be seen that our estimates for $\gamma$ are very close to the predicted value of $43 / 32$. Our estimates of $\gamma_{N T}$ are also quite close to this value, but they appear to be slightly and consistently lower than it. However, since they are so close, it is highly probable that they are in fact the same, and there is a slight systematic error in our fits.

Next, we estimate the value of $\nu_{N T}$. We calculate this exponent by fitting a straight line to $\log \left\langle R^{2}\right\rangle_{n}^{N T}$ against $\log n$ and estimating the slope of the line (see figure 8). As before, we found that we needed a correction-to-scaling term of $n^{-1}$ for both SAWs and 


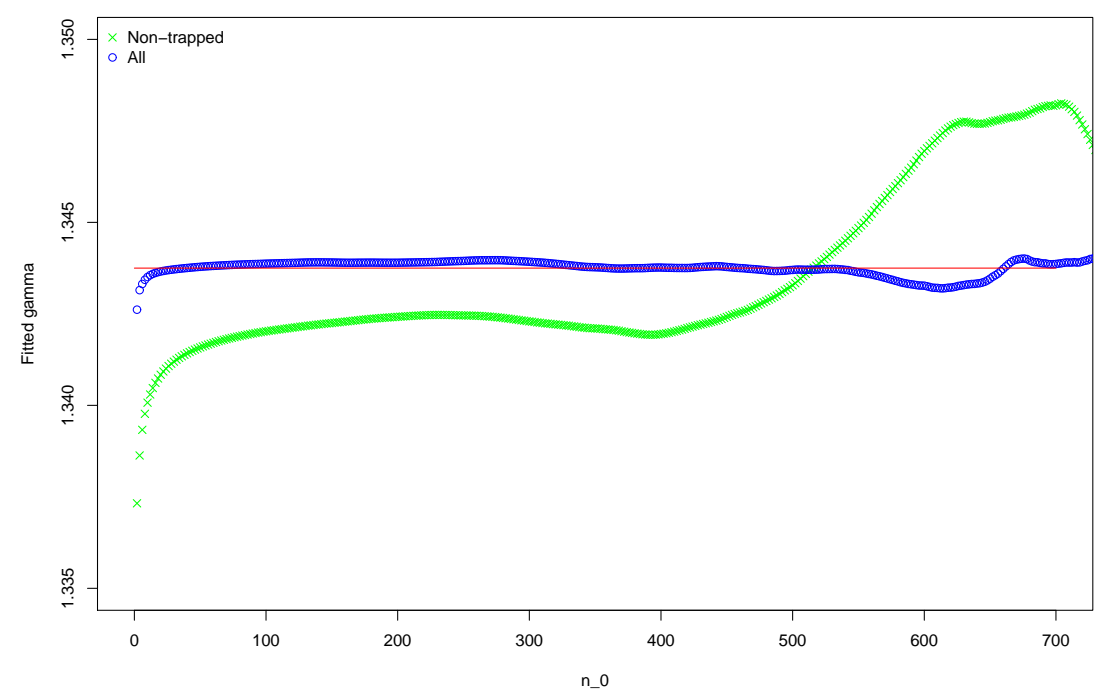

Figure 7. Fitted $\gamma_{N T}$ against $n_{0}$ for the square lattice, using a $n^{-1}$ correction term over a fitting window of $\left[n_{0}, \infty\right)$. The fits get much worse when fewer points are used.

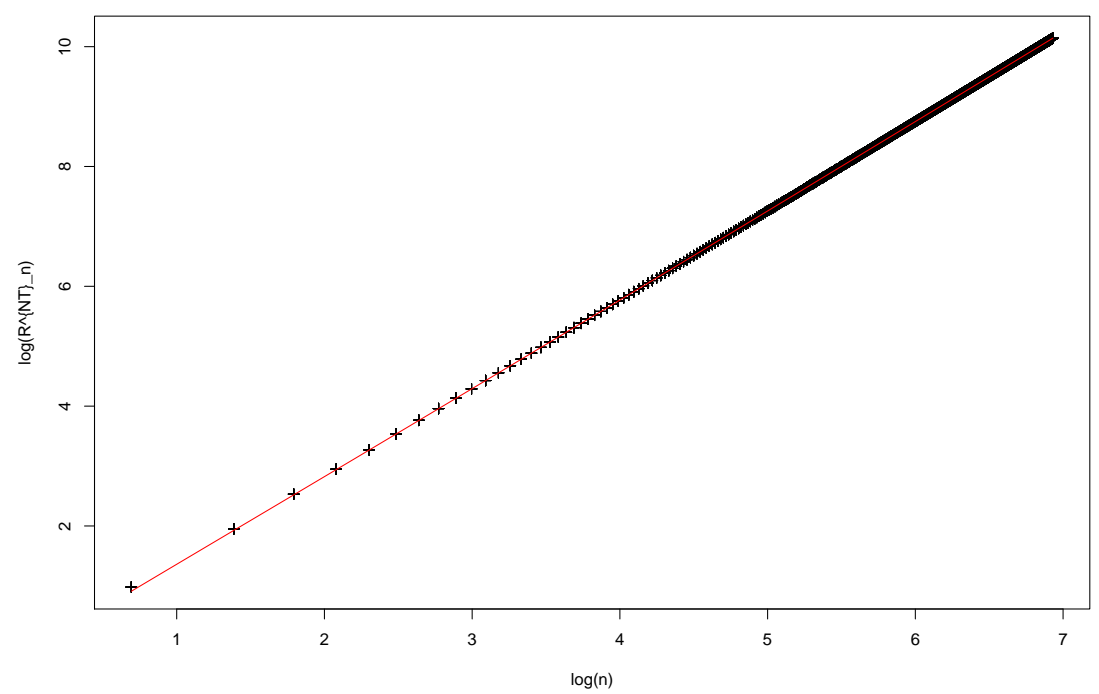

Figure 8. Log-log plot of end-to-end distance against $n$ for the square lattice, with fitted values for $n_{0}=100$.

NTSAWs. Figure 9 shows the corrected $\nu$ estimates against $n_{0}$.

We averaged our fits over the same intervals as the previous section, deriving the results in table 1 . Again, our estimates for $\nu$ are very close to the predicted value of $3 / 4$, whereas our estimates of $\nu_{N T}$ are slightly below this value. Once again, it is most probable that this is due to a small systematic error in our fits. 


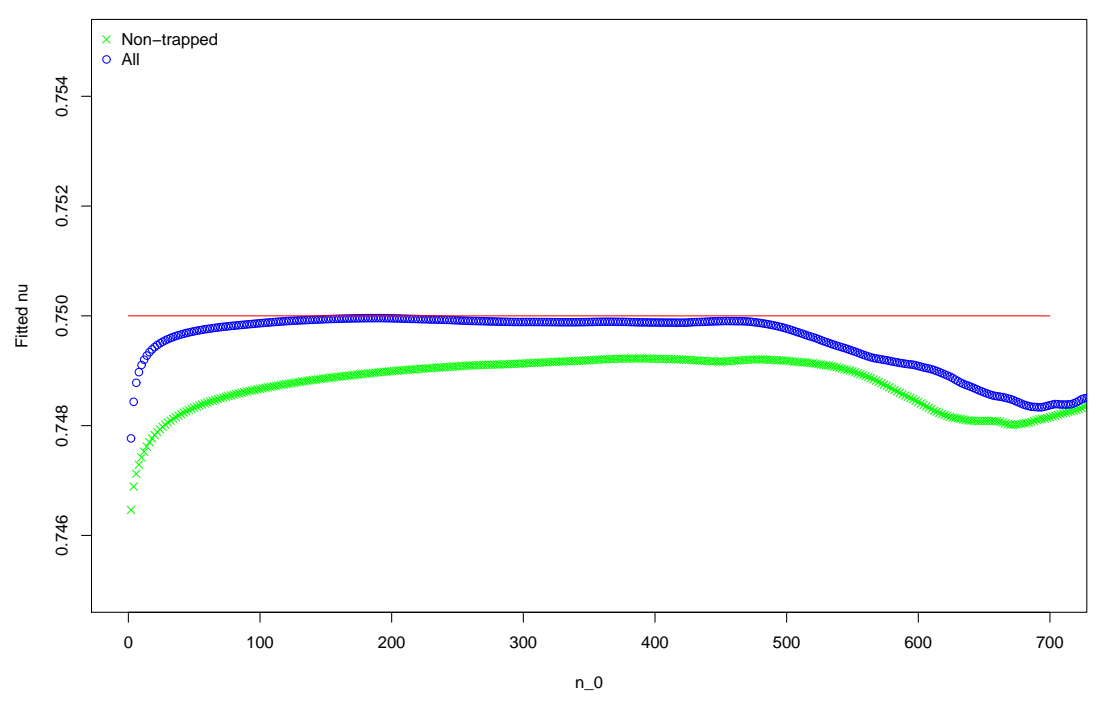

Figure 9. Fitted $\nu_{N T}$ against $n_{0}$ for the square lattice, using a $n^{-1}$ correction term over a fitting window of $\left[n_{0}, \infty\right)$. The fits get much worse when fewer points are used.

\subsection{Ratios}

In order to further examine the relationship between $\gamma$ and $\gamma_{N T}$, we now take another approach and make a direct comparison of $c_{n}^{N T}$ and $c_{n}$. The ratio of these numbers should grow as

$$
\frac{c_{n}^{N T}}{c_{n}}=\frac{A_{N T}}{A} n^{\gamma_{N T}-\gamma}(1+o(1))
$$

since they have the same growth rate. Hence if this ratio approaches a constant as $n \rightarrow \infty$, we can conclude that $\gamma_{N T}=\gamma$. Figure 10 shows a plot of this ratio against $n$, showing that this is indeed the case. We note that the ratios become nearly linear when we plot against $n^{-1 / 2}$; this allows us to estimate the limiting ratio using simple linear regression. This is shown in figure 11.

Fitting linear fits to a moving window of $n \in\left[n_{0}, \infty\right)$ do not indicate a trend with $n_{0}$, so we need not use a correction term. Our final estimates are averaged over $n_{0} \in[50,400]$ and are

$$
\begin{aligned}
& A_{N T}^{s q r} / A^{s q r}=0.9541(3), \\
& A_{N T}^{t r i} / A^{t r i}=0.9650(4), \quad A_{N T}^{\text {hex }} / A^{\text {hex }}=0.9440(2) .
\end{aligned}
$$

Here the numbers in brackets are the standard error of our estimates. Our estimates indicate that these ratios are not universal and that SAWs are most likely to trap themselves on the hexagonal lattice, which is not surprising.

We note that there is a significant difference between the proportions of trapped walks using our definition (4.6\%) and the definition of walks with zero atmosphere (calculated in [10] to be $0.9 \%$ ). Typically a walk is trapped several steps before it has zero atmosphere, and our results are consistent with this. 


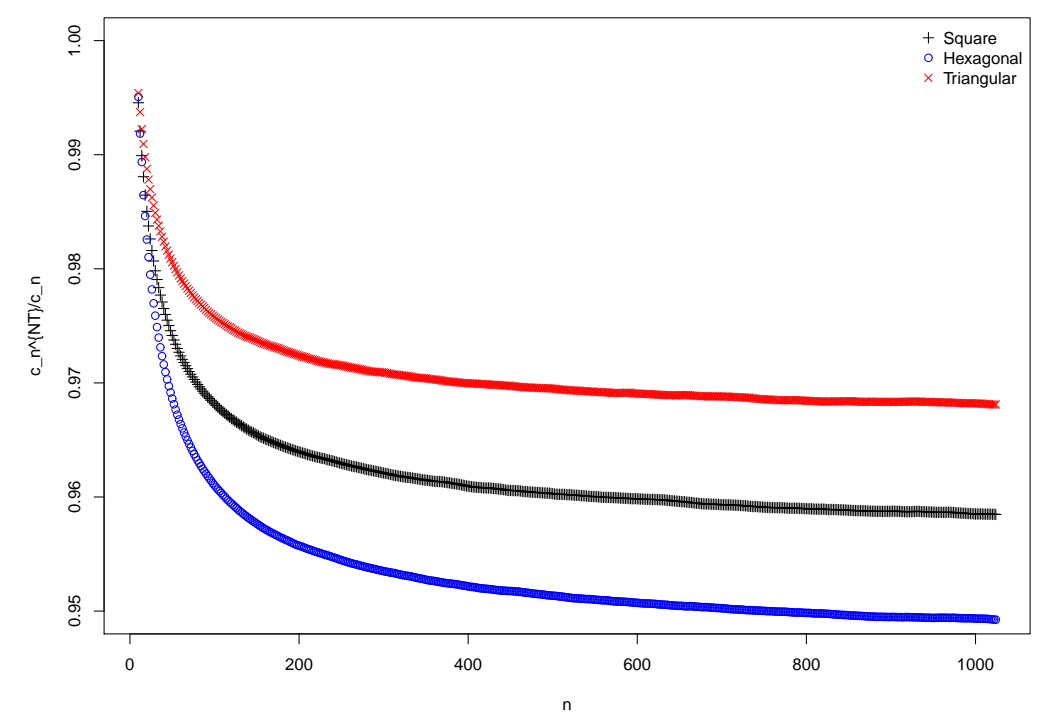

Figure 10. Ratios of non-trapped SAWs to all SAWs against $n$, for all lattices.

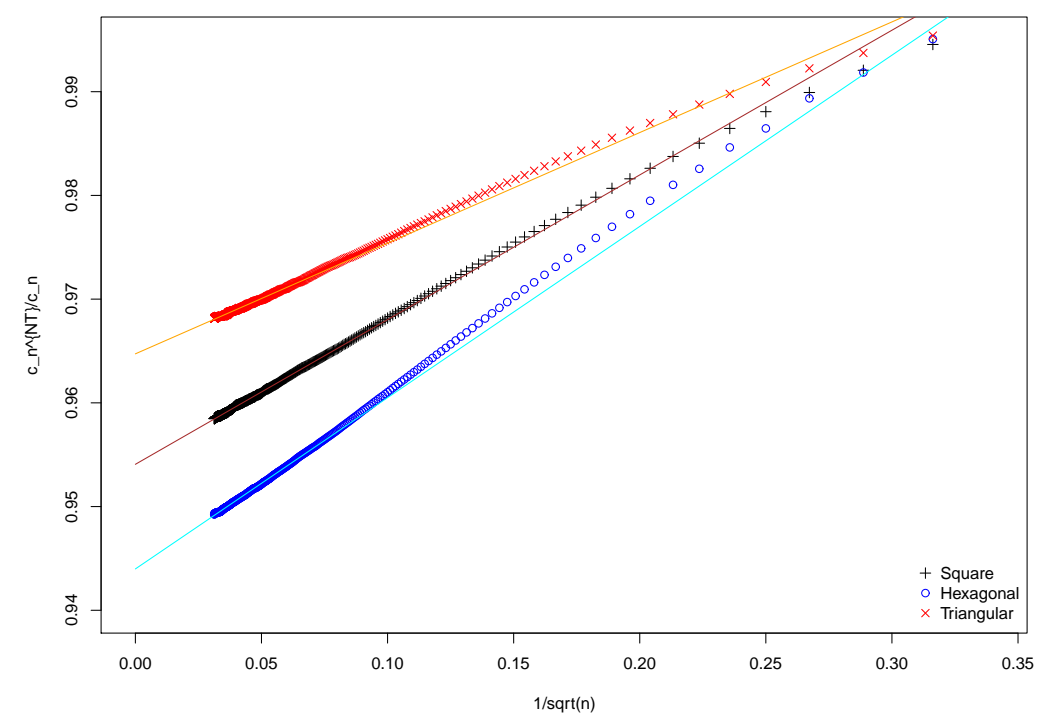

Figure 11. Ratios against $1 / \sqrt{n}$ with fitted lines, for all lattices.

We also looked at the ratio of squared end-to-end distances for non-trapped to all SAWs. This ratio should grow as

$$
\frac{\left\langle R^{2}\right\rangle_{n}^{N T}}{\left\langle R^{2}\right\rangle_{n}}=\frac{\alpha_{N T}}{\alpha} n^{2\left(\nu_{N T}-\nu\right)}(1+o(1))
$$

and if it approaches a constant as $n \rightarrow \infty$, we can again conclude that $\nu_{N T}=\nu$. It appears that this is also the case (see figure 12). When we plot the ratio against $n^{-1 / 2}$, we see linear behaviour only close to our largest values of $n$. Because of this curvature, we use a fit with $n^{-1 / 2}$ and $n^{-1}$ terms, which results in much more stable results. Our 


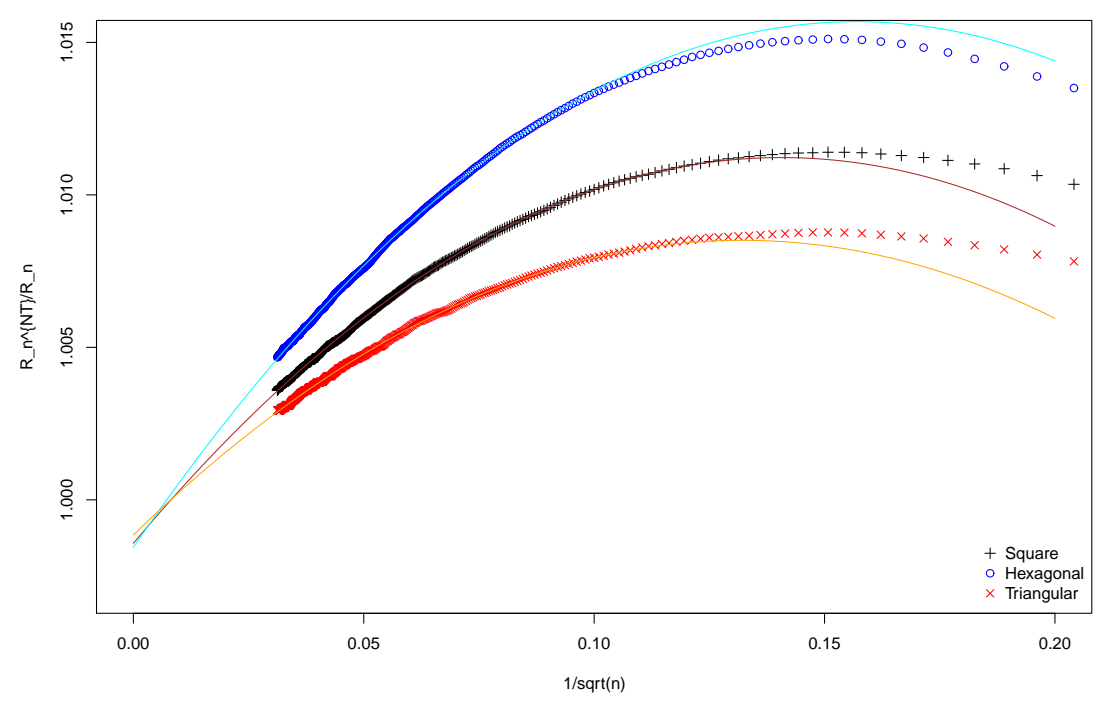

Figure 12. Ratio of end-to-end distances against $1 / \sqrt{n}$ with fitted curves using $n_{0}=100$, for all lattices.

final estimates are again averaged over $n_{0} \in[50,400]$ and are

$$
\begin{aligned}
& \alpha_{N T}^{s q r} / \alpha^{s q r}=0.9990(6), \\
& \alpha_{N T}^{t r i} / \alpha^{t r i}=0.9989(7), \quad \alpha_{N T}^{h e x} / \alpha^{\text {hex }}=0.9988(5) .
\end{aligned}
$$

These numbers are quite close to 1 (and remain close even if we try different fitting terms) and it seems reasonable to suppose that they are exactly 1 . Thus the intuition of non-trapped walks being "more expanded" than all walks turns out to be false, in the dominant asymptotic term at least.

If the ratios are exactly $1,(4)$ then implies that the ratio of squared end-to-end distances for trapped to all SAWs also tends to 1 (and thus has the same critical exponent). This follows because the proportions of the number of non-trapped and trapped walks to all SAWs tend to positive constants which sum to 1.

\subsection{Non-analytic correction-to-scaling term}

The ratio of the number of walks seems quite clearly to be linear against $n^{-1 / 2}$ (in particular plotting against $n^{-1}$ shows a clear curve for large $n$ ). This suggests the presence of a non-analytic correction-to-scaling term of $n^{-1 / 2}$ in the asymptotic behaviour of non-trapped self-avoiding walks. If this is so, this would be qualitatively different to all self-avoiding walks, where the first non-analytic correction-to-scaling term is $n^{-3 / 2}$. With this in mind, we re-visit our fits from section 3.1 to include this term. Therefore we fit to the form

$$
\log \frac{c_{n}^{N T}}{\mu^{n}}=\log A+\left(\gamma_{N T}-1\right) \log n+\frac{B}{n}+\frac{C}{n^{1 / 2}} .
$$




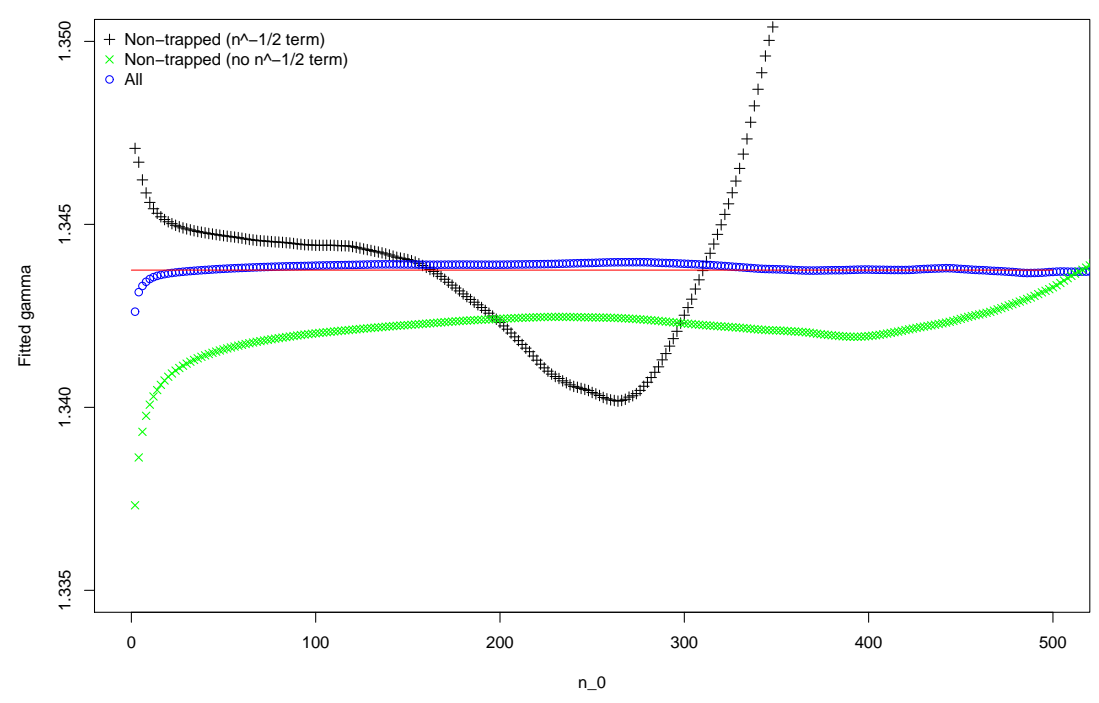

Figure 13. Fitted $\gamma_{N T}$ against $n_{0}$ for the square lattice, using correction terms over a fitting window of $\left[n_{0}, \infty\right)$. The fits get much worse when fewer points are used.

Table 2. Estimates of $\gamma_{N T}$ and $\nu_{N T}$ for various lattices, using a $n^{-1 / 2}$ correction-toscaling term. The numbers in brackets are the standard error of our estimates.

\begin{tabular}{lrr}
\hline Lattice & $\gamma_{N T}$ & $\nu_{N T}$ \\
\hline Square & $1.3444(11)$ & $0.75081(28)$ \\
Triangular & $1.3446(13)$ & $0.75056(48)$ \\
Hexagonal & $1.3449(13)$ & $0.75026(26)$ \\
\hline
\end{tabular}

After introducing this extra term, we found that the fits were less stable at larger $n_{0}$ (see figure 13). Because of this, we average our estimates over the smaller interval $n_{0} \in[50,150]$ and the results are shown in table 2 . We can see now that although the error-bars are larger, the estimates are closer to 43/32 (and indeed, this value is included in our error-bars). Given the evidence of the previous section, we consider this fit more trustworthy, and strong evidence that $\gamma_{N T}=\gamma$. Of course, we cannot rule out the possibility of very subtle differences in exponents, but we believe this to be unlikely.

It is less clear that the ratio of end-to-end distances is asymptotically linear in $n^{-1 / 2}$. While figure 12 suggests that this may be so, the ratio looks reasonably linear when plotted against a number of different terms (though not $n^{-1}$, which shows definite curvature for high $n$ ). Because $n^{-1 / 2}$ in particular works well for the ratio of the number of walks, we include it in our fits for $\left\langle R^{2}\right\rangle_{n}^{N T}$ (see figure 14). This results in the form

$$
\log \left\langle R^{2}\right\rangle_{n}^{N T}=\log A+2 \nu_{N T} \log n+\frac{B}{n}+\frac{C}{n^{1 / 2}}
$$

The results are also given in table 2 . Again, these values are more variable, but are closer to and include the SAW value of $3 / 4$. Once again we consider this strong evidence that 


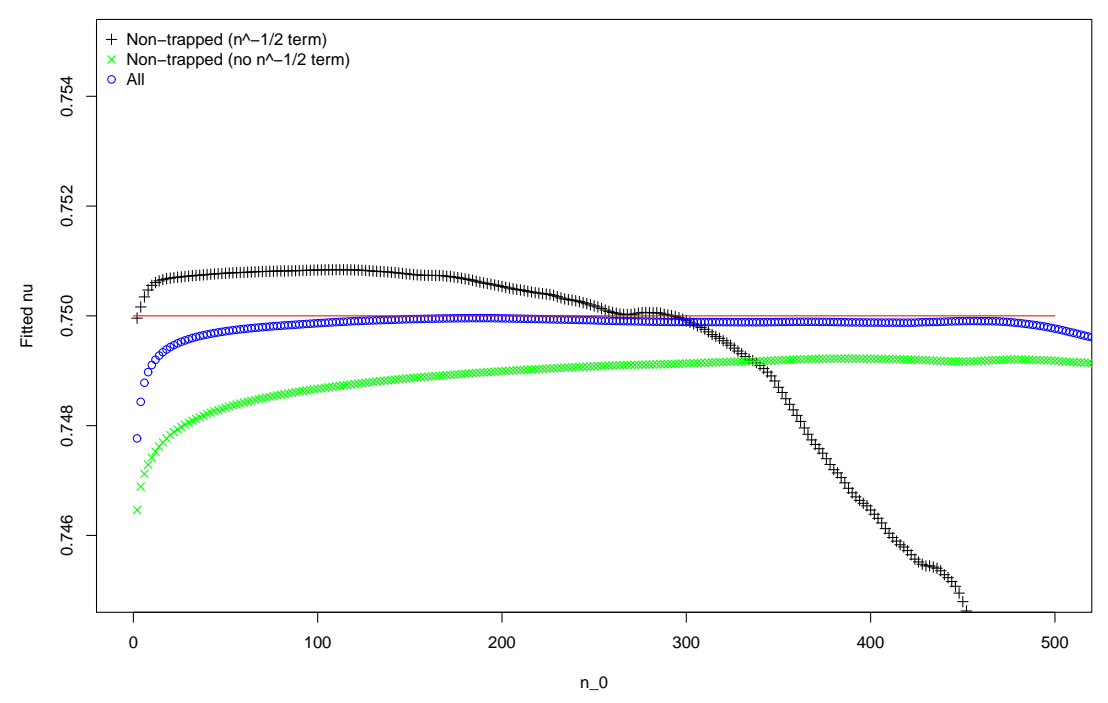

Figure 14. Fitted $\nu_{N T}$ against $n_{0}$ for the square lattice, using correction terms over a fitting window of $\left[n_{0}, \infty\right)$. The fits get much worse when fewer points are used.

the metric exponents are equal.

We note that the fits in this section are sufficiently variable that they do not constitute overwhelming evidence in favour of a $n^{-1 / 2}$ correction-to-scaling term for non-trapped SAWs. Since such a term is not present in the scaling of all SAWs, it follows that if it exists for non-trapped SAWs, then a corresponding cancelling term must exist for trapped SAWs. However, our plots and the quality of our fits provide quite compelling evidence that $c_{n}^{N T} / c_{n}$ is actually linear in $n^{-1 / 2}$.

\section{Conclusion}

In this paper, we have generated non-trapped self-avoiding walks of length up to 1024 by means of the flatPERM algorithm, on the square, triangular and honeycomb lattices. The results indicate that the critical exponent of the number of walks, $\gamma$, and the exponent of the mean squared end-to-end distance, $\nu$, are equal to their values for all self-avoiding walks, and in particular that they are still universal across the three lattices. This also implies that they are equal to their values for the alternative definition [10] of non-trapped walks as walks which can be continued by at least one step.

We have also estimated the limiting proportion of non-trapped walks to all selfavoiding walks, and the limiting ratio of their end-to-end distances. The former is a non-universal quantity, while the latter appears to be universally equal to 1 . Our findings also suggest the presence of a non-analytic correction-to-scaling term of $n^{-1 / 2}$, which is not present for all SAWs.

There are two interesting extensions of this work that we would like to pursue in the future. The first would be to determine whether or not the behaviour of non-trapped 
walks remains similar to that of SAWs when interactions are present. For example, one can ask if NTSAWs have the same free energy as all SAWs when adsorbing or collapsing. If this is the case, then one could take advantage of the non-trapping condition to simulate far longer conformations than is possible for SAWs and so probe further into low temperature regimes.

The second is to determine if the behaviour of NTSAWs and SAWs remain the same when they are sampled purely kinetically — this is equivalent to sampling walks with the Rosenbluth method, but all walks have weight 1 . It is conjectured [15] that the squared end-to-end distance of SAWs in this regime grows like $n^{4 / 3}$, but no corresponding conjecture has been made for NTSAWs.

\section{Acknowledgments}

We would like to acknowledge funding support from NSERC (AR), the ANR A3 project and the Austrian Science Foundation FWF, grant Z130-N13 (YBC). We thank Mireille Bousquet-Mélou for bringing this problem to our attention. We also thank Mireille and Tony Guttmann for helpful discussions and comments on the manuscript.

\section{References}

[1] Sergio Caracciolo, Anthony J. Guttmann, Iwan Jensen, Andrea Pelissetto, Andrew N. Rogers, and Alan D. Sokal. Correction-to-scaling exponents for two-dimensional self-avoiding walks. J. Stat. Phys., 120:1037-1100, 2005.

[2] Iwan Jensen. Enumeration of self-avoiding walks on the square lattice. J. Phys. A: Math. Gen., 37:5503-5524, 2004.

[3] Neal Madras and Gordon Slade. The self-avoiding walk. Birkhäuser, Boston, 1993.

[4] Iwan Jensen. A parallel algorithm for the enumeration of self-avoiding polygons on the square lattice. J. Phys. A: Math. Gen., 36:5731-5745, 2003.

[5] Mireille Bousquet-Mélou. Families of prudent self-avoiding walks. J. Combin. Theory Ser. A, 117:313-344, 2010.

[6] Mireille Bousquet-Mélou. On the importance sampling of self-avoiding walks. 2011. arXiv:1106.1876v1 [math.CO].

[7] Donald E. Knuth. Mathematics and computer science: coping with finiteness. Science, 194(4271):1235-1242, 1976.

[8] J. M. Hammersley. The number of polygons on a lattice. In Proc. Camb. Phil. Soc, volume 57, pages 516-523. Cambridge Univ. Press, 1961.

[9] J. M. Hammersley and D. J. A. Welsh. Further results on the rate of convergence to the connective constant of the hypercubical lattice. Q. J. Math., 13(1):108-110, 1962.

[10] Aleks Owczarek and Thomas Prellberg. Scaling of the atmosphere of self-avoiding walks. J. Phys. A: Math. Theor., 41:375004, 2008.

[11] Thomas Prellberg and Jarosław Krawczyk. Flat histogram version of the pruned and enriched Rosenbluth method. Phys. Rev. Lett., 92:120602, 2004.

[12] J. M. Hammersley and K. W. Morton. Poor man's Monte Carlo. J. Roy. Stat. Soc. B Met., 16(1):23-38, 1954.

[13] Marshall N. Rosenbluth and Arianna W. Rosenbluth. Monte Carlo calculation of the average extension of molecular chains. J. Chem. Phys., 23:356-360, 1955. 
[14] Peter Grassberger. Pruned-enriched Rosenbluth method: Simulations of $\theta$ polymers of chain length up to 1000000 . Phys. Rev. E, 56:3682-3693, 1997.

[15] Imtiaz Majid, Naeem Jan, Antonio Coniglio, and H. Eugene Stanley. Kinetic growth walk: a new model for linear polymers. Phys. Rev. Lett., 52(15):1257-1260, 1984. 